Historic, Archive Document

Do not assume content reflects current scientific knowledge, policies, or practices. 



\section{FALL 1926}

\section{THE FOSS HEATON GLAD GARDENS}

\section{SHANNON CITY, IOWA}

All quotations prepaid, 6 at dozen rate, 25 at 100 rate. Cash with crder, or 25 percent cash and balance before shipment, or C. O. D. All bulbs are young, healthy, and true to name. Fall or Spring shipment, just as you prefer. All stock offered subject to prior sale.

The 1927 GLAD GUIDE, with over 7,000 words of descriptive matter, will be mailed to you about January 1st, listing other varieties. But it would be well to malie your selections for next year from this list, as some varieties may be sold out at these prices.

Special quotations on quantities and iots gladly made. Special cor1espondence cordially invited.

No. 1 size means $1 \frac{1 / 2}{2}$ inch and sver. L means large bulbs, 11/4 inch and over. M. means medium size bulbs, between $3 / 1$. inch and $1 \frac{1}{1} /$ inch, averaging about one inch. S. means small bulbs, under $3 / 4$ inch. Bulblets that are priced by the dozen are selected.

\begin{tabular}{|c|c|c|c|c|c|c|c|}
\hline \multirow{4}{*}{$\begin{array}{l}\text { A. B. KUNDERD } \\
\text { Cream and pink, ruffled } \\
\text { ALLEN V. BUNCE }\end{array}$} & & & & & & \multicolumn{2}{|l|}{ Per } \\
\hline & Each & $\$ .08$ & $\$ .06$ & $\$$ & $s$ & 100 & $\$ .40$ \\
\hline & Doz. & .75 & .60 & .40 & .25 & & \\
\hline & Each & 1.00 & .75 & & & 12 & .59 \\
\hline Orange-Yellow Prim. & & & & & & 100 & 3.50 \\
\hline ALICE TIPLADY & Each & .05 & & & & 1000 & .75 \\
\hline Orange-Saffron Prim. & Doz. & .50 & .40 & & & & \\
\hline & 100 & 2.50 & 2.00 & 1.20 & .75 & & \\
\hline AMERICAN BEAUTY & Each & .12 & .10 & .06 & & & \\
\hline American Beauty Rose & Doz. & 1.20 & 1.00 & .60 & .30 & 100 & .25 \\
\hline & 109 & & & 4.50 & 2.50 & 1000 & 1.50 \\
\hline ANNA EBERIUS & Each & .06 & .05 & & & 100 & .15 \\
\hline Bright Purple & Doz. & .63 & .50 & & & 1000 & .75 \\
\hline & 100 & 3.75 & 3.00 & 2.00 & .75 & & \\
\hline ARABIA - - & Each & 1.00 & .75 & & & & \\
\hline Almost Black & $\frac{\sqrt{0 z}}{\sqrt{00}}$ & & & 20.00 & 15.00 & & \\
\hline VALON & Each & .03 & .06 & & & 100 & .20 \\
\hline Light Pink, Ruffled & Doz. & .75 & .60 & & & 1000 & 1.50 \\
\hline BREAK O' DAY & Each & 4.00 & 3.00 & 2.00 & 1.00 & Each & .15 \\
\hline Early Pink & & & & & & 12 & 1.50 \\
\hline & & & & & & 100 & 10.00 \\
\hline CAPT. BOYNTON & Each & .25 & .20 & .10 & .05 & 12 & .20 \\
\hline Lavender & Doz. & 2.50 & 2.00 & 1.00 & .50 & 100 & 1.00 \\
\hline & 100 & & & 7.50 & 3.50 & & \\
\hline ARDINAL PRINCE & Each & 1.00 & .75 & & & Each & .10 \\
\hline Cardinal Red & & & & & & 12 & 1.00 \\
\hline CLEOPATRA & Each & 1.00 & .75 & & & & \\
\hline Giant Yellow & & & & & & & \\
\hline CRIMSON GLOW & Each & .05 & & & & 1000 & .53 \\
\hline Crimson & Doz. & .50 & .40 & .20 & & & \\
\hline & 100 & 2.50 & 2.25 & 1.50 & & & \\
\hline CRINKLES & Each & .25 & .20 & & & 12 & .20 \\
\hline Dark Pink Ruffled & & & & & & & \\
\hline DR. ELKINS _-_.-.-. & Each & .20 & .15 & & & 12 & .29 \\
\hline White, Purple Blotch & Doz. & 2.00 & 1.50 & & & 100 & .75 \\
\hline DR. F. E. BENNETT --- & Each & .50 & .35 & .25 & .15 & 12 & .25 \\
\hline Bright Scarlet & Doz. & 5.00 & 3.50 & 2.50 & 1.50 & 100 & 1.50 \\
\hline & 100 & & & 20.00 & 12.00 & & \\
\hline DR. NELSON SHOOK & Each & 1.00 & .75 & & & Each & .10 \\
\hline Bright Magenta & & & & & & 12 & 1.00 \\
\hline EUCHESSE OF YORK & Each & & .15 & .06 & & 12 & .20 \\
\hline Blua Purple & Doz. & & 1.20 & .60 & .40 & 100 & 1.09 \\
\hline & 100 & & & 4.75 & 2.75 & & \\
\hline E. J. SHAYLOR & Each & .05 & & & & 100 & .20 \\
\hline Deep Rose & Doz. & .50 & & & & 1000 & 1.00 \\
\hline & 100 & 3.75 & 3.00 & 2.00 & 1.00 & & \\
\hline JLF & Each & & .05 & & & 1000 & .50 \\
\hline White, Canary Blotch & Doz. & & .50 & & & & \\
\hline & 100 & & 3.00 & 1.50 & .75 & & \\
\hline ELIZABETH TABOR & Each & .20 & .15 & & .05 & 103 & .40 \\
\hline Early Pink, Large & Doz. & 2.00 & 1.50 & & .50 & & \\
\hline FVELYN KIRTLAND & Each & & .05 & & & 1000 & .50 \\
\hline Salmon Pink & Doz. & & .40 & .20 & & & \\
\hline & 100 & & 2.00 & 1.00 & .50 & & \\
\hline $\begin{array}{l}\text { GERALDINE FARRAR } \\
\text { Light Blue }\end{array}$ & Each & & & 2.50 & 1.50 & & \\
\hline $\begin{array}{l}\text { Light Blue } \\
\text { GIANT MYRTLE }\end{array}$ & Each & .20 & .15 & .10 & .05 & 100 & (5) \\
\hline $\begin{array}{l}\text { GIANT MYRTLE } \\
\text { Pink and White, fine }\end{array}$ & Doz. & 2.00 & 1.50 & 1.00 & .50 & 100 & $. \partial i\}$ \\
\hline $\begin{array}{l}\text { Pink and White, fine } \\
\text { GIANT NYMPH }\end{array}$ & Each & .15 & .12 & .08 & & 12 & .15 \\
\hline Cream Pink & Doz. & 1.20 & 1.20 & .75 & & 100 & .75 \\
\hline GOLD & Each & .15 & .12 & & & 12 & .15 \\
\hline Light Yellow & Doz. & 1.50 & 1.20 & & & & \\
\hline GOLDEN BUTTERFLY & Each & .15 & .10 & .08 & & 100 & .25 \\
\hline Giant Yellow Prim. & Doz. & 1.50 & 1.00 & .75 & .40 & & \\
\hline GOLDEN FRILLS -..-- & Each & & 5.00 & & & Each & .40 \\
\hline Yellow Prim., Ruffled & & & & & & 12 & 4.00 \\
\hline GOLDEN MEASURE --. & Each & .08 & .06 & & & 100 & .20 \\
\hline Tall Light Yellow & Doz. & .75 & .60 & & & & \\
\hline & 100 & 6.00 & 5.00 & & & & \\
\hline HENRY FORD & Each & .25 & .20 & .15 & .10 & 12 & .20 \\
\hline Purple & Doz. & 2.50 & 2.00 & 1.50 & 1.00 & 100 & .75 \\
\hline & 100 & & & 12.03 & 7.50 & 1000 & 7.00 \\
\hline
\end{tabular}


HERADA Each Mauve

HIGHLAND LADDIE Each

NDIAN MAID Each $\quad 2.50$

Peach Blossom Prim.

Doz. $\quad .75$

J. A. CARBONE Orange-Salmon

JENNY LIND -

Each

Apricot Pink

Doz

WELL

Salmon Prim.

Doz.

TIS Doz.

Mahogany-Brow
Man T. PIRIE 100

K's YELLOW WONDER Each Light Yellow

I.EOTA Large Pink Doz.

Each LOS ANGELES LOUISE Lavender AIDEN'S BLUSH _..._... Each Pink Prim.

MARIE KUNDERD _- Each Ruffled White

MARSHALL FOCH _.... Each

Geranium Pink Doz

100

$\begin{array}{cc}\text { ONA LISA } & \text { Each } \\ \text { Light Lavender, Ruffled } & \text { Doz. }\end{array}$

Doz.

MRS. H.

Each $\quad .06$

Pink Scarlet Blotch

Doz.
100

MRS. LEON DOUGLAS _-_ Each Giant Beronia Rose

Doz.

IRS. FRANK PENDLETON _ _. Each Pink, Red Blotch

RS. F. C. PETERS Doz. $\quad .50$ Rose Lilac

IRS. L. S. SCHWEPPE _____ Each Peach Blossom Pink

$\quad .12$

W. H. PHIPPS Doz. 3.00

Giant Pink

OLA NEGRI _._. Each Apricot

FURPLE GLORY Doz.

Ruffled Dar

100

URPLE PERFECTION _-_._ Each Purple, Self-Color Doz.

RED FIRE -_._. Each Self-Colored Red

RICHARD DIENER _.___._. Each Melting Pink

ROBT. J. KUNDERD Deep red

ROSE ASH

Old Rose

Doz.

Doz.

ROSE GLORY _._._Each Rose, Ruffled

POSEMARY

Each Lavender Prim.

SCARLET WONDER _..... Each Large Red

EILA Dach Best Salmon Each
Doz. का

SOUVENIR Deep Yellow Prim Doz

$\begin{array}{llr}\text { Deep Yellow Prim } & \text { Doz. } \\ \text { SUPERBA } & 100 & 5.00 \\ \text { Each } & .30\end{array}$ Each $\quad .30 \quad .25$ 